OPEN ACCESS

Edited by:

John Whitelock,

University of New South Wales,

Australia

Reviewed by:

Jasmeen S. Merzaban,

King Abdullah University of Science

and Technology, Saudi Arabia

Nikos Karamanos,

University of Patras, Greece

*Correspondence:

Fredrik Noborn

Fredrik.Noborn@gu.se

Specialty section:

This article was submitted to

Signaling,

a section of the journal

Frontiers in Cell and Developmental

Biology

Received: 15 April 2021

Accepted: 27 July 2021

Published: 13 August 2021

Citation:

Noborn F, Nikpour M, Persson A,

Nilsson $J$ and Larson $G$ (2021)

Expanding the Chondroitin Sulfate

Glycoproteome - But How Far?

Front. Cell Dev. Biol. 9:695970.

doi: 10.3389/fcell.2021.695970

\section{Expanding the Chondroitin Sulfate Glycoproteome - But How Far?}

\author{
Fredrik Noborn ${ }^{1 *}$, Mahnaz Nikpour ${ }^{1}$, Andrea Persson ${ }^{1}$, Jonas Nilsson ${ }^{1,2}$ and \\ Göran Larson ${ }^{1}$
}

\begin{abstract}
1 Department of Laboratory Medicine, Institute of Biomedicine, Sahlgrenska Academy at the University of Gothenburg, Gothenburg, Sweden, ${ }^{2}$ Proteomics Core Facility, Sahlgrenska Academy at the University of Gothenburg, Gothenburg, Sweden
\end{abstract}

Chondroitin sulfate proteoglycans (CSPGs) are found at cell surfaces and in connective tissues, where they interact with a multitude of proteins involved in various pathophysiological processes. From a methodological perspective, the identification of CSPGs is challenging, as the identification requires the combined sequencing of specific core proteins, together with the characterization of the CS polysaccharide modification(s). According to the current notion of CSPGs, they are often considered in relation to a functional role in which a given proteoglycan regulates a specific function in cellular physiology. Recent advances in glycoproteomic methods have, however, enabled the identification of numerous novel chondroitin sulfate core proteins, and their glycosaminoglycan attachment sites, in humans and in various animal models. In addition, these methods have revealed unexpected structural complexity even in the linkage regions. These findings indicate that the number and structural complexity of CSPGs are much greater than previously perceived. In light of these findings, the prospect of finding additional CSPGs, using improved methods for structural and functional characterizations, and studying novel sample matrices in humans and in animal models is discussed. Further, as many of the novel CSPGs are found in low abundance and with not yet assigned functions, these findings may challenge the traditional notion of defining proteoglycans. Therefore, the concept of proteoglycans is considered, discussing whether "a proteoglycan" should be defined mainly on the basis of an assigned function or on the structural evidence of its existence.

Keywords: proteoglycans, glycosaminoglycans, chondroitin sulfate, core proteins, glycoproteomics, attachment site

\section{INTRODUCTION}

The concept of chondroitin sulfate proteoglycans (CSPGs) as discrete molecular entities first emerged during the late 1950s. At that time, chondroitin sulfate (CS) and protein complexes had been identified in hyaline cartilage, but the nature of the complexes remained elusive, and it was unclear whether the CS to protein association involved a covalent bond or not (Shatton and Schubert, 1954). In a pioneering study by Helen Muir, she showed that the CS chains were indeed covalently linked to the protein counterpart (Muir, 1958). A few years later, it was found that the CS polysaccharides are attached to serine residues of the core proteins via a tetrasaccharide linkage region, composed of Glucuronic acid (GlcA)-Galactose (Gal)-Galactose (Gal)-Xylose (Xyl) (Lindahl and Roden, 1966; Roden and Smith, 1966). Since then, an increasing number of different 
CSPGs have been identified, each distinguished by the primary sequence of the core protein and with different numbers of CS chains attached (Olson et al., 2006; Iozzo and Schaefer, 2015; Pomin and Mulloy, 2018; Toledo et al., 2020).

CSPGs are important components in connective tissue and fine-tunes a wide range of cellular processes, including neural development, growth factor signaling and inflammation (Hatano and Watanabe, 2020; Hussein et al., 2020). However, the structural identification of CSPGs is often difficult, as the identification requires the combined sequencing of specific core proteins together with the structural verification of any potential CS polysaccharides. Consequently, studies on identifying novel CSPGs, using earlier established biochemical techniques, were mostly focused on the characterization of single core proteins in a defined cellular or physiological context (Krusius and Ruoslahti, 1986; Fisher et al., 1989). Such techniques are typically based on different read out assays following enzymatic depolymerization with bacterial lyases and/or site directed mutagenesis of cloned proteins.

The lack of suitable analytical methods for large-scale analyses of CSPGs in biological samples has for a long time limited the ability to identify both novel CSPGs and assess the degree of heterogeneity of the CS glycoproteome across various systems.

This mini review will specifically focus on the recent advances in glycoproteomics to identify and characterize CSPGs in complex sample mixtures in humans and animal model systems. These methods include trypsin digestion, enrichment of acidic glycopeptides by strong anion-exchange chromatography (SAX), and incubation with chondroitinase $\mathrm{ABC}$ to reduce the length and complexity of the CS chains. The samples are then analyzed with reversed phase nano-liquid chromatographytandem mass spectrometry (nLC-MS/MS) and evaluated by glycopeptide search algorithms, resulting in the discovery and characterization of several novel CSPGs both in vertebrates and invertebrates. In light of these findings, the structural and conceptual insights that can be provided by such attachment sitespecific analysis of the CS glycoproteome are discussed. Further, as many of the novel CSPGs are found in low abundance and with no yet assigned functions, these findings may challenge the traditional notion of proteoglycans, in which the described proteoglycans often have an assigned function. Finally, the concept of the CS glycoproteome is discussed in relation to whether the glycoproteomic space should be regarded as a static or a dynamic entity.

\section{A GLYCOPROTEOMIC APPROACH TO IDENTIFY NOVEL PROTEOGLYCANS}

In glycobiology, mass spectrometry-based strategies for investigating protein glycosylation (glycoproteomics) have become an increasingly important tool (Nilsson et al., 2009; Thaysen-Andersen et al., 2016; Narimatsu et al., 2018). Such strategies, well-covered in excellent reviews, are typically based on enrichment of glycopeptides and subsequent analysis with nLC-MS/MS to provide site-specific information of $N$ - and O-glycans (Nilsson, 2016; Darula and Medzihradszky, 2018;
Madsen et al., 2020; Chernykh et al., 2021). To further develop this concept, we established a glycoproteomic protocol for global characterization of CS-glycopeptides in human urine and cerebrospinal fluid (CSF) (Noborn et al., 2015; Noborn et al., 2021). At first, bikunin (also known as protein AMBP) was used as a model CSPG since it was relatively well characterized, available in large quantities from human urine and also used in some countries as a pharmaceutical agent to treat acute pancreatitis (Ly et al., 2011; Lord et al., 2020). Human bikunin has a single CS chain of 27-39 monosaccharides attached to the N-terminal end (Ser-10) of the core protein (Lord et al., 2013). We incubated pharmaceutical grade bikunin with chondroitinase $\mathrm{ABC}$ that generated free disaccharides and a residual hexasaccharide structure still attached to the core protein. Digestion with trypsin generated a defined CS-glycopeptide suitable in size for nLC-MS/MS analysis. The analysis enabled the identification of several specific glycosidic and bikunin peptide fragments, serving as a proofof-concept that site-specific analysis of CSPGs is indeed a feasible strategy. We then enriched trypsin-digested CSPGs from human urine and CSF and incubated the enriched samples with chondroitinase $\mathrm{ABC}$, and thereafter analyzed the resulting CSglycopeptides by nLC-MS/MS. Generated data were evaluated through proteomic software with adjustments to allow for glycopeptide identification, enabling the identification of 13 novel human CSPGs in addition to 13 already established CSPGs. In Tables 1A,B, the novel proteoglycans identified in humans and different model systems through glycoproteomics are shown. Interestingly, several of the novel human CSPGs were traditionally defined as prohormones, which was surprising, as prohormones are not typically regarded to belong to the proteoglycan superfamily (Noborn et al., 2015). This approach demonstrates the structural and conceptual insights that can be provided by global attachment site-specific analysis of the CS glycoproteome.

A similar strategy was developed for complex proteoglycans of the extracellular matrix (hyalectans) from human and bovine sources (Klein et al., 2018). In addition to CS chains, hyalectan proteoglycans are also rich $N$ - and mucin-type $O$-glycosylations that decorate large parts of the proteins. The abundant glycosylation impairs effective peptide identification and reduces the overall sequence coverage achieved by mass-spectrometric based methods. By using a combination of efficient enrichment procedures for glycosylated peptides and advanced MS/MSsoftware analysis, the authors were able to improve the peptide sequence coverage of these complex proteoglycans and identify a number of new attachment sites, including both $N_{-}$, mucin type $\mathrm{O}$ - and CS-glycosylations.

\section{GLYCOPROTEOMICS: NOVEL CSPGS LEADING TO NOVEL HYPOTHESES}

Although glycoproteomic approaches assist in identifying novel CSPGs, the functional relevance of a CS modification on cellular physiology is often unclear and cannot be inferred from only the CS structure or from the core protein alone. 
TABLE 1 | A. Novel human proteoglycans identified through glycoproteomics.

\begin{tabular}{|c|c|c|c|c|}
\hline \multicolumn{5}{|l|}{ Human } \\
\hline Brain-specific angiogenesis inhibitor 2 & 060241 & CS & $266^{\star}$ & Noborn et al., 2015 \\
\hline CD99L2 protein & $\mathrm{HOY} 4 \mathrm{H} 3$ & CS & $141^{*}$ & Noborn et al., 2015 \\
\hline Cholecystokinin & P06307 & CS & 31 & Noborn et al., 2015 \\
\hline Collagen and calcium-binding EGF domain containing protein 1 & Q6UXH8 & CS & 385 & Noborn et al., 2015 \\
\hline Laminin subunit gamma 2 & Q13753 & CS & $803^{*}$ & Toledo et al., 2020 \\
\hline Matrix-remodeling associated protein 5 & Q9NR99 & CS & $702^{*}$ & Noborn et al., 2015 \\
\hline Meprin A subunit alpha & Q16819 & CS & $631^{*}$ & Nasir et al., 2016 \\
\hline Natriuretic peptides B & P16860 & CS & $41^{*}$ & Toledo et al., 2020 \\
\hline Neurexin-1 & Q9ULB1 & HS & 1,355 & Zhang et al., 2018 \\
\hline Nidogen-2 & Q14112 & CS & $452^{\star}, 358^{\star}$ & Toledo et al., 2020 \\
\hline Osteopontin & P10451 & CS & $234^{\star}, 308^{*}$ & Noborn et al., 2015 \\
\hline Plexin domain-containing protein 1 & Q8IUK5 & CS & 33 & Noborn et al., 2015 \\
\hline Retinoic acid responder protein & P49788 & CS & 40 & Nasir et al., 2016 \\
\hline Secretogranin-1 & P05060 & CS & $93^{*}, 239^{*}$ & Noborn et al., 2015 \\
\hline Secretogranin-3 & Q8WXD2 & CS & 37 & Noborn et al., 2015 \\
\hline Sushi repeat-containing protein SRPX & P78539 & CS & $34^{\star}$ & Toledo et al., 2020 \\
\hline
\end{tabular}

B. Novel proteoglycans identified in different model systems through glycoproteomics.

\begin{tabular}{lccc}
\hline Protein name & $\begin{array}{c}\text { Uniprot ID/Accession } \\
\text { number }\end{array}$ & $\begin{array}{c}\text { Modification } \\
b\end{array}$ & ${\text { Site }(\mathbf{s})^{b}}^{\text {References }}$ \\
\hline
\end{tabular}

\section{Caenorabditis elegans}

CLE-1A protein/CPG-10

COLlagen/CPG-11

C-type lectin domain containing protein 180/CPG-12

Dauer Up-Regulated, isoform b/CPG-13

High Incidence of Males, isoform b/CPG-14

LiPocalin-Related protein/CPG-15

FiBrillin homolog/CPG-16

Papilin/CPG-17

Protein C45E5.4/CPG-18

Protein C06G1.2/CPG-19

Protein K08B4.2/CPG-20

Protein R17.3/CPG-21

Protein T10E9.3/CPG-22

Protein Y39B6B.y/CPG-23

Protein Y41D4B.26/CPG-24

Danio rerio

Uncharacterized protein si:ch73-306e8.2 isoform X1

Drosophila melanogaster

Protein Windpipe

Rat INS-1 832/13, murine MIN-6 and NIT-1 cell lines

Chromogranin-A

Immunoglobulin superfamily member 10

Islet Amyloid Polypeptide

Secretogranin-1

Q9U9K7
Q22651
Q19970
Q7JLY2
NCBINP_001024582.1
Q23163
Q23587
O76840
Q18642
Q17742
Q91YY6
O18003
O01603
NCBI PIR T45051
Q8WSN8
XP_00133717.3
Q9W266
P10354
Q3V1M1
P12969
P35314

$\begin{array}{ccc}\text { Chn } & 581 & \text { Noborn et al., 2018 } \\ \text { Chn } & 336^{*} & \text { Noborn et al., 2018 } \\ \text { Chn } & 275,290 & \text { Noborn et al., 2018 } \\ \text { Chn } & 368 & \text { Noborn et al., 2018 } \\ \text { Chn } & 4,850^{*} & \text { Noborn et al., 2018 } \\ \text { Chn } & 172^{*} & \text { Noborn et al., 2018 } \\ \text { Chn } & 1,079^{*} & \text { Noborn et al., 2018 } \\ \text { Chn } & 775^{*} & \text { Noborn et al., 2018 } \\ \text { Chn } & 38^{*} & \text { Noborn et al., 2018 } \\ \text { Chn } & 35^{*} & \text { Noborn et al., 2018 } \\ \text { Chn } & 167^{*} & \text { Noborn et al., 2018 } \\ \text { Chn } & 111^{*} & \text { Noborn et al., 2018 } \\ \text { Chn } & 382,399 & \text { Noborn et al., 2018 } \\ \text { Chn } & 313^{*} & \text { Noborn et al., 2018 } \\ \text { Chn } & 194 & \text { Noborn et al., 2018 } \\ & & \\ \text { CS } & 103^{*} & \text { Delbaere et al., 2020 } \\ & & \\ \text { CS } & 282,335,337 & \text { Takemura et al., 2020 } \\ \text { HS } & 433 & \text { Nikpour et al., 2021 } \\ \text { CS } & 679 & \text { Nikpour et al., 2021 } \\ \text { CS } & 28 & \text { Nikpour et al., 2021 } \\ \text { HS } & 236 & \text { Nikpour et al., 2021 } \\ & & \end{array}$

aGlycosaminoglycan modifications: CS, chondroitin sulfate; HS, heparan sulfate; or Chn, chondroitin.

${ }^{b}$ Glycosaminoglycan attachment sites denote serine amino acid numbers, determined unambiguously by nLC-MS/MS or ambiguously for glycopeptides containing two or more serine residues $\left({ }^{*}\right)$. Numbers relate to the corresponding protein ID. 
However, the identification of novel CSPGs, as well as defining novel attachment site modifications of established CSPGs, generate novel hypotheses that can be experimentally tested. Here we exemplify with a few cases of how such global or targeted glycoproteomic approaches can be combined with hypothesis-driven research to investigate structure-function relationships of CSPGs.

We recently identified the Windpipe protein as a novel CSPGs in Drosophila melanogaster and found that the core protein carried three separate CS chains in its extracellular domain (Takemura et al., 2020). Genetic engineering combined with morphological analyses demonstrated that Windpipe inhibited Hedgehog (Hh) signaling in a CS dependent manner. Interestingly, Windpipe-overexpression resulted in reduced wing size compared with control flies. However, the wing size was restored in genetically modified flies in which all the three CS attachment serine residues were substituted with alanine residues (thereby precluding CS modification). This study thus demonstrates a novel role of a specific CSPG in regulating $\mathrm{Hh}$ signaling and illustrate the potential of combining glycoproteomics with molecular and cell biological studies in a well-defined model system.

Several protein hormones are stored as amyloid-like aggregates in the secretory granules of endocrine cells (Maji et al., 2009). Most of the protein hormones require mild acidic $\mathrm{pH}$ and the addition of low molecular weight heparin or CS for their aggregation in vitro. Our finding that several prohormones carry CS chains lead us to hypothesize that the CS side chains may facilitate self-assembly of the prohormones (Noborn et al., 2015). Indeed, binding studies showed that CS promoted the assembly of the chromogranin A core protein under acidic condition, giving a possible explanation to previous observations that chromogranin A has an inherent property to assemble in the acidic milieu of secretory granules. Whether the CS side chains of other prohormones (e.g., secretogranin-1 and 3) may also influence the assembly of their respective core protein remains to be determined.

Furthermore, an affinity-enriched glycoproteomic approach was recently employed to explore the involvement of CSPGs in the pathogenesis of pregnancy-associated malaria. The disease is caused by the parasite Plasmodium falciparum and has potentially very severe clinical outcome for both mother and child (Toledo et al., 2020; Tomlinson et al., 2020). The parasite induces expression of the malarial protein VAR2CSA on the surface of infected erythrocytes, which enables their binding to structural variants of CS in the placental intervillous space of pregnant women (Kane and Taylor-Robinson, 2011). This interaction is dependent on the size and sulfation-type of the polysaccharide, as longer chains with higher degree of C4-O- sulfation of GalNAc residues display stronger binding (Sugiura et al., 2016; Ma et al., 2021). A VAR2CSA-affinity column was recently used to enrich for CSPGs, capable of binding the VAR2CSA-protein, in human placenta. The enriched fraction was then analyzed through a CS glycoproteomic workflow, showing that a collection of different core proteins, rather than a single core protein, carried CS chains that were capable of binding the VAR2CSA-protein (Toledo et al., 2020). This indicates that several different CSPGs may serve as attachment factors for malaria-infected erythrocytes, which provides new insight into the disease etiology. Notably, CS and heparan sulfate (HS) also constitute attachment factors for many enveloped and non-enveloped viruses (Olofsson and Bergström, 2005). Interestingly, recent studies show that cell surface HS act as a co-receptor to ACE2 and is essential for SARS-CoV-2 infection (Clausen et al., 2020; Mycroft-West et al., 2020). To our knowledge, however, the information of a potential HS core protein (or group of core proteins) involved in such processes is scarce (Daly et al., 2020). A glycoproteomic enrichment strategy, similar to the VAR2CSA-CS enrichment approach, may provide information on this issue.

\section{PROTEOGLYCAN LINKAGE REGION COMPLEXITY}

The CS linkage region has previously been perceived as a relatively uniform entity with limited structural variability. MS/MS-analysis of chondroitinase ABC-digested bikunin described a defined hexasaccharide structure with $O$-sulfations of the GalNAc residue and of the outer Gal residue (Ly et al., 2011). In addition to sulfation, other linkage region modifications have been reported, e.g., sialylation and phosphorylation (Sugahara et al., 1988; Kitagawa et al., 2008; Lu et al., 2010; Wen et al., 2014). However, the reports have been limited to only a few separate experimental systems and information on potential combinations of linkage region modifications is still scarce. To this aim, our glycoproteomic analysis of human bikunin and other proteoglycans from urine, CSF, and plasma have revealed an unexpected linkage region complexity, with different combinations of sulfation, phosphorylation and sialylation (Gomez Toledo et al., 2015; Noborn et al., 2015; Nasir et al., 2016; Nilsson et al., 2017). Furthermore, the structural variability of human bikunin was further increased by large variations in the mucin type $O$-linked glycosylation found nearby the CS site (Gomez Toledo et al., 2015). Moreover, an unexpected fucose (deoxy-hexose) modification was found on the xylose residue of the linkage region of human bikunin and decorin and on two novel human CSPGs; retinoic acid responder protein 1 and meprin A (Gomez Toledo et al., 2015; Nasir et al., 2016). This was surprising as fucosylated CS chains have previously only been described in sea cucumbers, a member of the phylum of Echinodermata (Myron et al., 2014; Dwivedi and Pomin, 2020). Notably, this fucose modification in human CS was located on xylose (and not on the CS chain), i.e., close to the protein component. This position is similar to the fucose modifications on $N$-glycans, which also may occur at the innermost GlcNAc residue linked to Asn in the consensus amino acid sequence (core fucosylation) (Plomp et al., 2017; Schneider et al., 2017; Wang and Ravetch, 2019). This kind of $N$-glycan fucosylation occurs both in invertebrate and vertebrate species and modifies the functional effects of the corresponding proteins, e.g., by regulating the inflammatory immune responses by modifying the $N$-glycans of the Fc chains of IgG1 molecules. This is of major importance when designing and introducing monoclonal antibodies for immune therapy, since the afucosylated variants show an increased antibody-dependent cellular cytotoxicity (ADCC) (Pereira et al., 2018). What functional effects the 
core fucosylation of xylose of CSPG may have remains to be determined.

Furthermore, we have observed a non-canonical linkage region trisaccharide (GlcA-Gal-Xyl-O-) on glycopeptides of bikunin from urine of healthy donors. This trisaccharide thus lacks one Gal residue of the traditional linkage region, and results in a residual pentasaccharide structure upon chondroitinase ABC digestion (Persson et al., 2019). Interestingly, mutations in the B3GALT6 gene, which codes for galactosyltransferase II ( $\beta 3 \mathrm{GalT} 6)$ is associated with Ehlers-Danlos-like syndromes, characterized by a spectrum of skeletal and connective tissue "linkeropathy" disorders (Malfait et al., 2013). The B3GalT6 enzyme is responsible for adding the second Gal residue in the GAG linkage region and mutations in the gene results in reduced GAG biosynthesis and appearance of the trisaccharide linkage region in a zebrafish b3galt6 knock-out model (Delbaere et al., 2020). Whether patients with B3GALT6 mutations also have increased levels of the non-canonical trisaccharide linkage region of bikunin in their urine remains to be determined. Taken together, at least 14 different variants of the bikunin linkage region (including canonical and non-canonical sequences) have now been described, thus demonstrating a much greater variability than previously appreciated (Gomez Toledo et al., 2015; Noborn et al., 2015; Nilsson et al., 2017; Persson et al., 2019). Xylose phosphorylation has been shown to function as a molecular switch to regulate the proteoglycan biosynthesis (Wen et al., 2014). The removal of xylose phosphorylation by 2-phosphoxylose phosphatase is considered a prerequisite for the polymerization of the linkage regions into longer chains (Koike et al., 2014). However, the influence of the other linkage modifications (or the combinations thereof) on the downstream GAG biosynthesis is yet unknown. Nevertheless, knowledge of linkage region complexity provides a theoretical framework for future functional-structural studies of CS biosynthesis. Moreover, analysis of extended site-specific CS chains, in theory achieved by using partial chondroitinase $\mathrm{ABC}$ digestion, may assist in further understanding of how these modifications affect the biosynthesis and thus the final structures of CS. In addition, this may provide information on how certain core protein determinants (e.g., amino acid sequences) may influence the CS structures. The concept of core protein specific structures have been difficult to address since most established proteoglycan workflows involve the separation of the CS polysaccharides and the core proteins. Ideally, site-specific analysis of full-length CS structures is desirable, as this type of information is likely required to fully explore the structural-functional basis of CSPGs.

Further development of the glycoproteomic concept has enabled site-specific characterization of both CS and HS sites (Noborn et al., 2016). To this aim, perlecan (also known as basement membrane specific proteoglycan) was chosen as a model proteoglycan since it is known to be substituted with both CS and HS chains (Iozzo, 2005; Noborn et al., 2015). A trypsin-digested perlecan sample, derived from EngelbrethHolm-Swarm mouse sarcoma, was enriched on a SAX column and digested with heparinase or chondroitinase $\mathrm{ABC}$, generating residual tetrasaccharide and hexasaccharide structures still attached to the peptide. This allowed for the identification of a glycopeptide derived from the N-terminal domain of perlecan, which encompassed the three previously known HS sites (Iozzo and Sanderson, 2011). In addition, the combination of chondroitinase and heparinase digestion revealed a "hybrid site" in the C-terminal domain, carrying either an HS or a CS chain (Noborn et al., 2016). This was surprising since it demonstrated that a single GAG attachment site is capable of carrying either HS or CS chains, thus, revealing a less appreciated level of proteoglycan heterogeneity. The identification of such "hybrid sites" is likely to be biologically important as HS and CS often display opposite effects on cellular physiology (Coles et al., 2011; Doody et al., 2015). Furthermore, similar glycoproteomic protocols have been used to identify novel HSPGs, and to investigate the functional role of HSPGs in neuronal synapse formation (Zhang et al., 2018; Montoliu-Gaya et al., 2021; Nikpour et al., 2021).

\section{EXPANDING THE PROTEOGLYCAN GLYCOPROTEOME - BUT HOW FAR?}

Glycosylation is one of the most ubiquitous forms of posttranslational modification and is expected to be present on more than half of all mammalian proteins (Apweiler et al., 1999; Schjoldager et al., 2020). However, despite its high abundance, GAG modifications are considered to occur only on a very minor proportion of the 20,000-25,000 proteins encoded by the human genome. Although glycoproteomic studies of various sample matrices have significantly increased our structural knowledge of proteoglycans, fewer than 80 core proteins carrying CS have so far been identified in humans (Noborn et al., 2015; Nasir et al., 2016; Toledo et al., 2020). A list of all CSPGs known to date in humans was recently published by Toledo et al. (2020). The number of human core proteins carrying HS are even less, and so far only a little more than 20 HSPGs have been established (Zhang et al., 2018; Nikpour et al., 2021). Moreover, while proteoglycans in vertebrates have been the focus of several structural studies, the information about proteoglycans in invertebrates is scarce and the reports are restricted to only a few species (Noborn and Larson, 2021). Thus, only a limited number of CSPGs have been identified in Drosophila melanogaster, which is surprising since it is one of the most studied invertebrates (Momota et al., 2011; Zhu et al., 2019; Takemura et al., 2020). Future studies employing glycoproteomic approaches will likely identify novel CSPGs of invertebrates and further expand our knowledge of the proteoglycan glycoproteome in different species.

The enzymatic transfer of a Xyl residue to certain Ser residues in the proteoglycan core protein initiates GAG biosynthesis (Briggs and Hohenester, 2018). The Xyl-modified Ser residue is typically followed by a Gly residue (-SG-) and is associated with a cluster of acidic residues in close proximity, indicating that certain motifs of the amino acid sequence influence the initiation process (Esko and Zhang, 1996). Such motifs may assist in the prediction of potential GAG sites of core proteins and provides insights into the potential space of the GAG glycoproteome. When we recently mapped the chondroitin (Chn) glycoproteome in C. elegans, this resulted in the identification of 15 novel core proteins in addition to the 9 previously established core 
proteins (Olson et al., 2006; Noborn et al., 2018). Bioinformatic analysis of protein sequences in the regions of the glycosylated Ser residues showed a highly stringent attachment motif E/DG/A-S-G. A search in the Swiss-Prot database using this motif retrieved 19 additional potential CPGs, indicating that possibly additional CPGs are yet to be found in the nematode (Noborn et al., 2018). However, it is unclear why these assumed CPGs escaped detection by the glycoproteomic analysis. This may simply relate to the sensitivity of the assay and that the glycopeptides corresponding to these potential CPGs were below the detection threshold. On the other hand, it may also suggest that some motifs are only occasionally occupied, or substituted with HS instead of Chn. Nevertheless, this demonstrates the ambiguity of using only attachment motifs as a strategy to identify proteoglycans, and that glycoproteomic analysis is needed to determine the GAG glycoproteome in any given sample system. The basic strategies for proteoglycan identification and the different levels of structural- and functional understanding of their roles in any biological system is schematically illustrated in Figure 1.

The prohormone chromogranin-A $(\mathrm{CgA})$ is one of the main proteins in secretory granules of endocrine cells and the precursor for several bioactive peptides (Kim et al., 2001). CgA has previously been established as a CSPG and carries a CS chain in the C-terminal end (Ser-424) of the human core protein (Gowda et al., 1990; Noborn et al., 2015). Our recent glycoproteomic analysis of cultured insulin-secreting cells, demonstrate that $\mathrm{CgA}$ in rodents, but not in humans, also carries

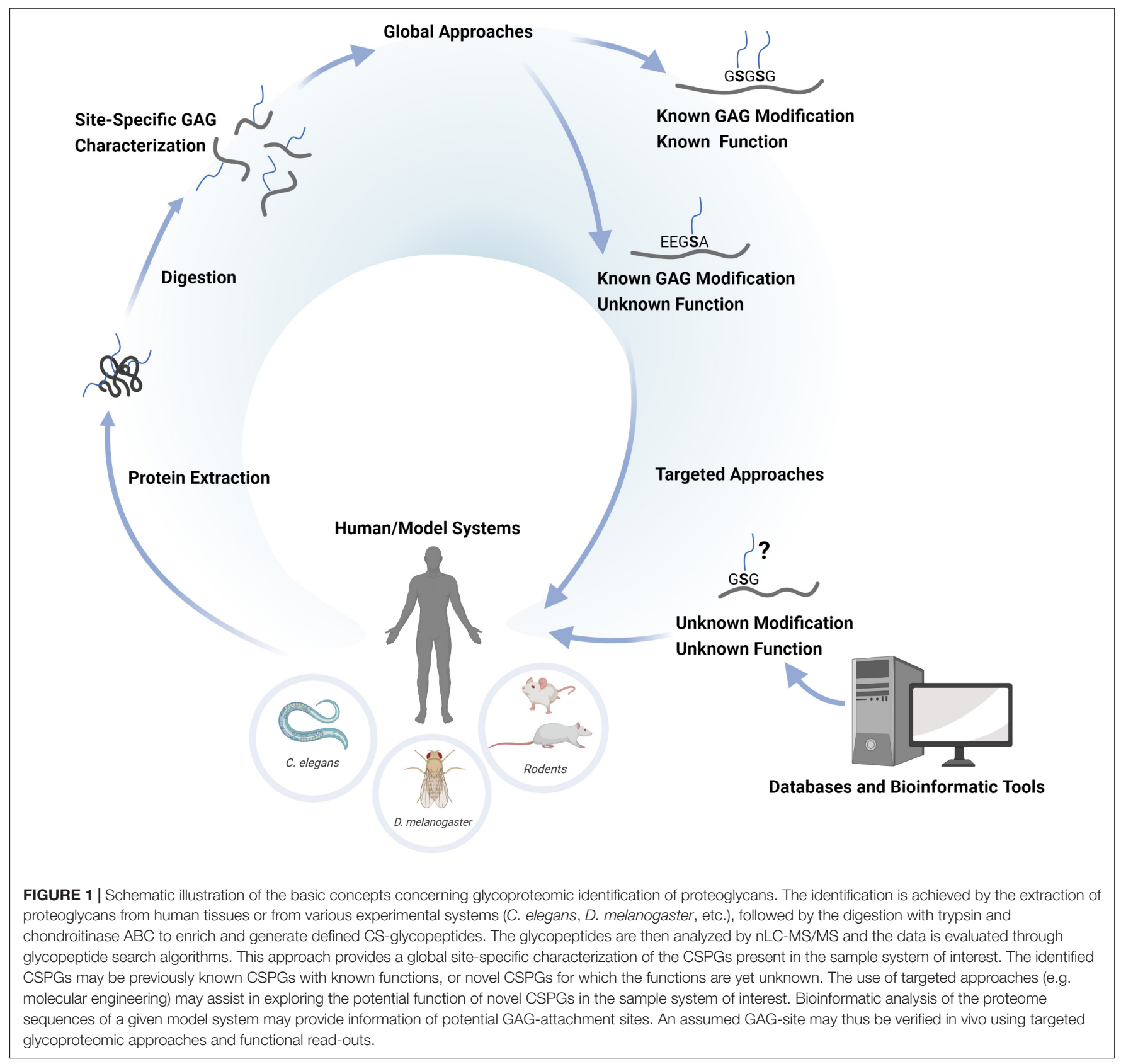


HS at the same C-terminal site, making this another "hybrid site" (Noborn et al., 2016; Nikpour et al., 2021). Although the glycosite is highly conserved between species (Noborn et al., 2015), this highlights the importance of cell-targeted mapping and that GAG modifications found in one cell type or tissue or species may not simply be inferred to another sample system. These findings also support the view that the GAG glycoproteome should not be regarded as a static entity, but rather a dynamic system that changes with the cellular physiology. A key issue for the future is to comprehend the biological factors that eventually will govern the expansion of the GAG glycoproteome.

\section{DISCUSSION}

As with any research area, the characterization of any particular biomolecule is critical for the understanding of its role in biological and pathological processes. The identification and structural characterization of proteoglycans is no exception. According to our current understanding of CSPGs, these are often regarded in a functional context, in which any given proteoglycan regulate a specific function in cellular pathophysiology. For example, decorin, a small leucine-rich extracellular matrix proteoglycan, is well-studied for its role as a suppressor of tumor cell growth (Neill et al., 2016; Appunni et al., 2019). Decorin binds and antagonizes various receptor tyrosine kinases, thereby inhibiting downstream oncogenic signaling and reducing tumor growth (Reszegi et al., 2020; Hu X. et al., 2021). Apart from their involvement in cancer, CSPGs are also in focus of other research areas, including neurogenesis and spinal cord injury (Maeda, 2015). Following damage to the central nervous system, axons fail to regenerate due to the formation of a glial scar, which is composed of extracellular matrix components including CSPGs (Hussein et al., 2020). The production of several CSPG family members is differentially regulated in the glial scar as neurocan, brevican and versican are increased, while aggrecan is reduced (Mukhamedshina et al., 2019). How the individual CSPGs contribute to the pathogenesis is yet unclear, but their relevance is illustrated by the interest of using chondroitinase $\mathrm{ABC}$ as a therapeutic agent (Bradbury et al., 2002; Hu et al., 2018; Hu J. et al., 2021). Although detailed structural-functional understanding of PGs many times remains unknown, the examples given in this review point to essential roles of CSPGs in various pathophysiological conditions and demonstrate the significant progress that has been made in the field in recent years.

The recent advances in glycoproteomics have enabled the identification of several novel CS core proteins in humans and in various animal models. With such techniques, additional CS core proteins and novel GAG modifications will likely be discovered with no yet assigned function/s. Thus, one should consider whether "a proteoglycan" should be defined mainly on the basis of an assigned function or on structural evidence of its existence. Importantly, glycoproteomic strategies have the potential of finding many novel proteoglycans and provide global structural information that may contribute to our conceptual understanding of the complex family of proteoglycans. However, viewing proteoglycans from a "structure only" perspective will meet its limitations. The development of even more advanced glycoproteomics strategies will presumably identify proteoglycans at low abundances and/or at low levels of occupancy. This suggests that caution is needed in the interpretation of the data as minute amounts of any given proteoglycan may have little or no relevance for our understanding of any given biological system. Therefore, using glycoproteomics in a clearly defined biological or pathological context will likely be a necessary future strategy, since it will set constraints on the interpretation of the structural information derived. This will likely assist in determining which proteoglycans are relevant for understanding the underlying biological mechanisms in any given system. Nonetheless, glycoproteomic strategies will surely assist in further expanding the knowledge of proteoglycan core proteins and their functions. In the perspective of the present pandemic of COVID-19, the importance of proteoglycans as attachment factors/receptors or co-receptors of pathogenic viruses cannot be underestimated and detailed structural information of the host GAGs and their core protein structures will most likely be warranted to understand the molecular details of virus infections as well as to design novel anti-viral drugs.

\section{AUTHOR CONTRIBUTIONS}

FN and GL did the writing and provided the main concepts discussed in this mini review. MN, AP, and JN assisted in the organization and editing of the publication and contributed to the table and the figure. All authors contributed to the article and approved the submitted version.

\section{FUNDING}

This review covers the recent advancement of glycoproteomic analysis of proteoglycans, and discusses the basis of site-specific glycan characterization, which has been an important concept in glycobiology during the last decade. As a principal investigator, GL has been conducting basic and applied research in this area and has been funded by several agencies during this time, and most lately from Vetenskapsrådet (Swedish Research Council 2017-00955) and Swedish Governmental grants to the Sahlgrenska University Hospital, Västra Götalandsregionen under the ALF-agreement (ALFGBG-721971). As a research team, we sincerely thank all these agencies, which are each listed in the respective publications which they supported.

\section{ACKNOWLEDGMENTS}

We thank Lena Kjellén and Paul O'Callaghan (Uppsala University) for valuable intellectual discussions. We also thank the proteomics Core Facility at the Sahlgrenska Academy, University of Gothenburg which performed most of the nLCMS/MS-analyses referenced in this manuscript. The illustration was created with Biorender.com. 


\section{REFERENCES}

Appunni, S., Anand, V., Khandelwal, M., Gupta, N., Rubens, M., and Sharma, A. (2019). Small Leucine Rich Proteoglycans (decorin, biglycan and lumican) in cancer. Clin. Chim. Acta 491, 1-7. doi: 10.1016/j.cca.2019 .01 .003

Apweiler, R., Hermjakob, H., and Sharon, N. (1999). On the frequency of protein glycosylation, as deduced from analysis of the SWISS-PROT database. Biochim. Biophys. Acta 1473, 4-8. doi: 10.1016/s0304-4165(99) 00165-8

Bradbury, E. J., Moon, L. D., Popat, R. J., King, V. R., Bennett, G. S., Patel, P. N., et al. (2002). Chondroitinase ABC promotes functional recovery after spinal cord injury. Nature 416, 636-640.

Briggs, D. C., and Hohenester, E. (2018). Structural Basis for the Initiation of Glycosaminoglycan Biosynthesis by Human Xylosyltransferase 1. Structure 26, 801-809.e3.

Chernykh, A., Kawahara, R., and Thaysen-Andersen, M. (2021). Towards structure-focused glycoproteomics. Biochem. Soc. Trans. 49, 161-186. doi: $10.1042 /$ bst20200222

Clausen, T. M., Sandoval, D. R., Spliid, C. B., Pihl, J., Perrett, H. R., Painter, C. D., et al. (2020). SARS-CoV-2 Infection Depends on Cellular Heparan Sulfate and ACE2. Cell 183, 1043-1057.e15.

Coles, C. H., Shen, Y., Tenney, A. P., Siebold, C., Sutton, G. C., Lu, W., et al. (2011). Proteoglycan-specific molecular switch for RPTPsigma clustering and neuronal extension. Science 332, 484-488. doi: 10.1126/science.12 00840

Daly, J. L., Simonetti, B., Klein, K., Chen, K. E., Williamson, M. K., Anton-Plagaro, C., et al. (2020). Neuropilin-1 is a host factor for SARS-CoV-2 infection. Science $370,861-865$.

Darula, Z., and Medzihradszky, K. F. (2018). Analysis of Mammalian O-Glycopeptides-We Have Made a Good Start, but There is a Long Way to Go. Mol. Cell Proteomics 17, 2-17. doi: 10.1074/mcp.mr117.00 0126

Delbaere, S., De Clercq, A., Mizumoto, S., Noborn, F., Bek, J. W., Alluyn, L., et al. (2020). b3galt6 Knock-Out Zebrafish Recapitulate B3GalT6Deficiency Disorders in Human and Reveal a Trisaccharide Proteoglycan Linkage Region. Front. Cell Dev. Biol. 8:597857. doi: 10.3389/fcell.2020.5 97857

Doody, K. M., Stanford, S. M., Sacchetti, C., Svensson, M. N., Coles, C. H., Mitakidis, N., et al. (2015). Targeting phosphatase-dependent proteoglycan switch for rheumatoid arthritis therapy. Sci. Transl. Med. 7:288ra276.

Dwivedi, R., and Pomin, V. H. (2020). Marine Antithrombotics. Mar. Drugs 18:514. doi: $10.3390 / \mathrm{md} 18100514$

Esko, J. D., and Zhang, L. (1996). Influence of core protein sequence on glycosaminoglycan assembly. Curr. Opin. Struct. Biol. 6, 663-670. doi: 10.1016/ s0959-440x $(96) 80034-0$

Fisher, L. W., Termine, J. D., and Young, M. F. (1989). Deduced protein sequence of bone small proteoglycan I (biglycan) shows homology with proteoglycan II (decorin) and several nonconnective tissue proteins in a variety of species. J. Biol. Chem. 264, 4571-4576. doi: 10.1016/s0021-9258(18)8 3781-4

Gomez Toledo, A., Nilsson, J., Noborn, F., Sihlbom, C., and Larson, G. (2015). Positive Mode LC-MS/MS Analysis of Chondroitin Sulfate Modified Glycopeptides Derived from Light and Heavy Chains of The Human Interalpha-Trypsin Inhibitor Complex. Mol. Cell Proteomics 14, 3118-3131. doi: 10.1074/mcp.m115.051136

Gowda, D. C., Hogue-Angeletti, R., Margolis, R. K., and Margolis, R. U. (1990). Chromaffin granule and PC12 cell chondroitin sulfate proteoglycans and their relation to chromogranin A. Arch. Biochem. Biophys. 281, 219-224. doi: 10. 1016/0003-9861(90)90435-2

Hatano, S., and Watanabe, H. (2020). Regulation of Macrophage and Dendritic Cell Function by Chondroitin Sulfate in Innate to Antigen-Specific Adaptive Immunity. Front. Immunol. 11:232. doi: 10.3389/fimmu.2020.00232

Hu, H. Z., Granger, N., Pai, S. B., Bellamkonda, R. V., and Jeffery, N. D. (2018). Therapeutic efficacy of microtube-embedded chondroitinase $\mathrm{ABC}$ in a canine clinical model of spinal cord injury. Brain 141, 1017-1027. doi: 10.1093/brain/ awy007
Hu, J., Rodemer, W., Zhang, G., Jin, L. Q., Li, S., and Selzer, M. E. (2021). Chondroitinase ABC Promotes Axon Regeneration and Reduces Retrograde Apoptosis Signaling in Lamprey. Front. Cell Dev. Biol. 9:653638. doi: 10.3389/ fcell.2021.653638

Hu, X., Villodre, E. S., Larson, R., Rahal, O. M., Wang, X., Gong, Y., et al. (2021). Decorin-mediated suppression of tumorigenesis, invasion, and metastasis in inflammatory breast cancer. Commun. Biol. 4:72. doi: 10.1201/b14586-5

Hussein, R. K., Mencio, C. P., Katagiri, Y., Brake, A. M., and Geller, H. M. (2020). Role of Chondroitin Sulfation Following Spinal Cord Injury. Front. Cell Neurosci. 14:208. doi: 10.3389/fncel.2020.00208

Iozzo, R. V. (2005). Basement membrane proteoglycans: from cellar to ceiling. Nat. Rev. Mol. Cell. Biol. 6, 646-656. doi: 10.1038/n rm1702

Iozzo, R. V., and Sanderson, R. D. (2011). Proteoglycans in cancer biology, tumour microenvironment and angiogenesis. J. Cell Mol. Med. 15, 1013-1031. doi: 10.1111/j.1582-4934.2010.01236.x

Iozzo, R. V., and Schaefer, L. (2015). Proteoglycan form and function: a comprehensive nomenclature of proteoglycans. Matrix Biol. 42, 11-55. doi: 10.1016/j.matbio.2015.02.003

Kane, E. G., and Taylor-Robinson, A. W. (2011). Prospects and Pitfalls of Pregnancy-Associated Malaria Vaccination Based on the Natural Immune Response to Plasmodium falciparum VAR2CSA-Expressing Parasites. Malar. Res. Treat. 2011:764845.

Kim, T., Tao-Cheng, J. H., Eiden, L. E., and Loh, Y. P. (2001). Chromogranin A, an "on/off" switch controlling dense-core secretory granule biogenesis. Cell 106, 499-509. doi: 10.1016/s0092-8674(01)00459-7

Kitagawa, H., Tsutsumi, K., Ikegami-Kuzuhara, A., Nadanaka, S., Goto, F., Ogawa, T., et al. (2008). Sulfation of the galactose residues in the glycosaminoglycan-protein linkage region by recombinant human chondroitin 6-O-sulfotransferase-1. J. Biol. Chem. 283, 27438-27443. doi: 10.1074/jbc. m803279200

Klein, J. A., Meng, L., and Zaia, J. (2018). Deep Sequencing of Complex Proteoglycans: a Novel Strategy for High Coverage and Site-specific Identification of Glycosaminoglycan-linked Peptides. Mol. Cell Proteomics 17, 1578-1590. doi: 10.1074/mcp.ra118.000766

Koike, T., Izumikawa, T., Sato, B., and Kitagawa, H. (2014). Identification of phosphatase that dephosphorylates xylose in the glycosaminoglycan-protein linkage region of proteoglycans. J. Biol. Chem. 289, 6695-6708. doi: 10.1074/ jbc.m113.520536

Krusius, T., and Ruoslahti, E. (1986). Primary structure of an extracellular matrix proteoglycan core protein deduced from cloned cDNA. Proc. Natl. Acad. Sci. U. S. A. $83,7683-7687$. doi: 10.1073/pnas.83.20.7683

Lindahl, U., and Roden, L. (1966). The chondroitin 4-sulfate-protein linkage. J. Biol. Chem. 241, 2113-2119. doi: 10.1016/s0021-9258(18) 96674-3

Lord, M. S., Day, A. J., Youssef, P., Zhuo, L., Watanabe, H., Caterson, B., et al. (2013). Sulfation of the bikunin chondroitin sulfate chain determines heavy chain.hyaluronan complex formation. J. Biol. Chem. 288, 22930-22941. doi: 10.1074/jbc.m112.404186

Lord, M. S., Melrose, J., Day, A. J., and Whitelock, J. M. (2020). The Inter-alpha-Trypsin Inhibitor Family: versatile Molecules in Biology and Pathology. J. Histochem. Cytochem. 68, 907-927. doi: 10.1369/0022155420 940067

Lu, H., Mcdowell, L. M., Studelska, D. R., and Zhang, L. (2010). Glycosaminoglycans in Human and Bovine Serum: detection of TwentyFour Heparan Sulfate and Chondroitin Sulfate Motifs Including a Novel Sialic Acid-modified Chondroitin Sulfate Linkage Hexasaccharide. Glycobiol. Insights 2010, 13-28. doi: 10.4137/gbi.s4273

Ly, M., Leach, F. E. III., Laremore, T. N., Toida, T., Amster, I. J., and Linhardt, R. J. (2011). The proteoglycan bikunin has a defined sequence. Nat. Chem. Biol. 7, 827-833. doi: $10.1038 /$ nchembio.673

Ma, R., Lian, T., Huang, R., Renn, J. P., Petersen, J. D., Zimmerberg, J., et al. (2021). Structural basis for placental malaria mediated by Plasmodium falciparum VAR2CSA. Nat. Microbiol. 6, 380-391. doi: 10.1038/s41564-020-00858-9

Madsen, T. D., Hansen, L. H., Hintze, J., Ye, Z., Jebari, S., Andersen, D. B., et al. (2020). An atlas of O-linked glycosylation on peptide hormones reveals diverse biological roles. Nat. Commun. 11:4033. 
Maeda, N. (2015). Proteoglycans and neuronal migration in the cerebral cortex during development and disease. Front. Neurosci. 9:98. doi: 10.3389/fnins.2015. 00098

Maji, S. K., Perrin, M. H., Sawaya, M. R., Jessberger, S., Vadodaria, K., Rissman, R. A., et al. (2009). Functional amyloids as natural storage of peptide hormones in pituitary secretory granules. Science 325, 328-332. doi: 10.1126/science. 1173155

Malfait, F., Kariminejad, A., Van Damme, T., Gauche, C., Syx, D., MerhiSoussi, F., et al. (2013). Defective initiation of glycosaminoglycan synthesis due to B3GALT6 mutations causes a pleiotropic Ehlers-Danlos-syndrome-like connective tissue disorder. Am. J. Hum. Genet. 92, 935-945. doi: 10.1016/j.ajhg. 2013.04.016

Momota, R., Naito, I., Ninomiya, Y., and Ohtsuka, A. (2011). Drosophila type $\mathrm{XV} / \mathrm{XVIII}$ collagen, Mp, is involved in Wingless distribution. Matrix Biol. 30, 258-266. doi: 10.1016/j.matbio.2011.03.008

Montoliu-Gaya, L., Tietze, D., Kaminski, D., Mirgorodskaya, E., Tietze, A. A., and Sterky, F. H. (2021). CA10 regulates neurexin heparan sulfate addition via a direct binding in the secretory pathway. EMBO Rep. 22:e51349.

Muir, H. (1958). The nature of the link between protein and carbohydrate of a chondroitin sulphate complex from hyaline cartilage. Biochem. J. 69, 195-204. doi: 10.1042/bj0690195

Mukhamedshina, Y. O., Povysheva, T. V., Nikolenko, V. N., Kuznecov, M. S., Rizvanov, A. A., and Chelyshev, Y. A. (2019). Upregulation of proteoglycans in the perilesion perimeter in ventral horns after spinal cord injury. Neurosci. Lett. 704, 220-228. doi: 10.1016/j.neulet.2019. 04.006

Mycroft-West, C. J., Su, D., Pagani, I., Rudd, T. R., Elli, S., Gandhi, N. S., et al. (2020). Heparin Inhibits Cellular Invasion by SARS-CoV-2: structural Dependence of the Interaction of the Spike S1 Receptor-Binding Domain with Heparin. Thromb. Haemost. 120, 1700-1715. doi: 10.1055/s-0040-17 21319

Myron, P., Siddiquee, S., and Al Azad, S. (2014). Fucosylated chondroitin sulfate diversity in sea cucumbers: a review. Carbohydr. Polym. 112, 173-178. doi: 10.1016/j.carbpol.2014.05.091

Narimatsu, H., Kaji, H., Vakhrushev, S. Y., Clausen, H., Zhang, H., Noro, E., et al. (2018). Current Technologies for Complex Glycoproteomics and Their Applications to Biology/Disease-Driven Glycoproteomics. J. Proteome Res. 17, 4097-4112. doi: 10.1021/acs.jproteome.8b 00515

Nasir, W., Toledo, A. G., Noborn, F., Nilsson, J., Wang, M., Bandeira, N., et al. (2016). SweetNET: a Bioinformatics Workflow for Glycopeptide MS/MS Spectral Analysis. J. Proteome Res. 15, 2826-2840. doi: 10.1021/acs.jproteome. $6 \mathrm{~b} 00417$

Neill, T., Schaefer, L., and Iozzo, R. V. (2016). Decorin as a multivalent therapeutic agent against cancer. Adv. Drug Deliv. Rev. 97, 174-185. doi: 10.1016/j.addr. 2015.10.016

Nikpour, M., Nilsson, J., Persson, A., Noborn, F., Vorontsov, E., and Larson, G. (2021). Proteoglycan profiling of human, rat and mouse insulin-secreting cells. Glycobiology doi: 10.1093/glycob/cwab035 [Epub Online ahead of print].

Nilsson, J. (2016). Liquid chromatography-tandem mass spectrometry-based fragmentation analysis of glycopeptides. Glycoconj. J. 33, 261-272. doi: 10.1007/ s10719-016-9649-3

Nilsson, J., Noborn, F., Gomez Toledo, A., Nasir, W., Sihlbom, C., and Larson, G. (2017). Characterization of Glycan Structures of Chondroitin SulfateGlycopeptides Facilitated by Sodium Ion-Pairing and Positive Mode LCMS/MS. J. Am. Soc. Mass Spectrom. 28, 229-241. doi: 10.1007/s13361-0161539- 1

Nilsson, J., Ruetschi, U., Halim, A., Hesse, C., Carlsohn, E., Brinkmalm, G., et al. (2009). Enrichment of glycopeptides for glycan structure and attachment site identification. Nat. Methods 6, 809-811. doi: 10.1038/nmeth. 1392

Noborn, F., Gomez Toledo, A., Green, A., Nasir, W., Sihlbom, C., Nilsson, J., et al. (2016). Site-specific identification of heparan and chondroitin sulfate glycosaminoglycans in hybrid proteoglycans. Sci. Rep. 6:34537.

Noborn, F., Gomez Toledo, A., Nasir, W., Nilsson, J., Dierker, T., Kjellen, L., et al. (2018). Expanding the chondroitin glycoproteome of Caenorhabditis elegans. J. Biol. Chem. 293, 379-389. doi: 10.1074/jbc.m117.80 7800
Noborn, F., Gomez Toledo, A., Sihlbom, C., Lengqvist, J., Fries, E., Kjellen, L., et al. (2015). Identification of chondroitin sulfate linkage region glycopeptides reveals prohormones as a novel class of proteoglycans. Mol. Cell Proteomics 14, 41-49. doi: $10.1074 /$ mcp.m114.043703

Noborn, F., and Larson, G. (2021). Characterization of C. elegans Chondroitin Proteoglycans and Their Large Functional and Structural Heterogeneity; Evolutionary Aspects on Structural Differences Between Humans and the Nematode. Adv. Exp. Med. Biol. 21, 155-170. doi: 10.1007/5584_2 020_485

Noborn, F. N., Persson, M., Sihlbom, A., Nilsson, C., and Larson, J. (2021). A Glycoproteomic Approach to Identify Novel Proteoglycans. Methods Mol. Biol. Glycosaminoglycans, 978-1-0716-1397-9, 481765_2_En, 2303, ed. K. Balagurunathan, et al.

Olofsson, S., and Bergström, T. (2005). Glycoconjugate glycans as viral receptors. Ann. Med. 37, 154-172. doi: 10.1080/07853890510007340

Olson, S. K., Bishop, J. R., Yates, J. R., Oegema, K., and Esko, J. D. (2006). Identification of novel chondroitin proteoglycans in Caenorhabditis elegans: embryonic cell division depends on CPG-1 and CPG-2. J. Cell Biol. 173, 985-994. doi: 10.1083/jcb.200603003

Pereira, N. A., Chan, K. F., Lin, P. C., and Song, Z. (2018). The "less-is-more" in therapeutic antibodies: Afucosylated anti-cancer antibodies with enhanced antibody-dependent cellular cytotoxicity. MAbs 10, 693-711. doi: 10.1080/ 19420862.2018.1466767

Persson, A., Nilsson, J., Vorontsov, E., Noborn, F., and Larson, G. (2019). Identification of a non-canonical chondroitin sulfate linkage region trisaccharide. Glycobiology 29, 366-371. doi: 10.1093/glycob/cwz014

Plomp, R., Ruhaak, L. R., Uh, H. W., Reiding, K. R., Selman, M., HouwingDuistermaat, J. J., et al. (2017). Subclass-specific IgG glycosylation is associated with markers of inflammation and metabolic health. Sci. Rep. 7:12325.

Pomin, V. H., and Mulloy, B. (2018). Glycosaminoglycans and Proteoglycans. Pharmaceuticals (Basel) 11:27.

Reszegi, A., Horvath, Z., Feher, H., Wichmann, B., Tatrai, P., Kovalszky, I., et al. (2020). Protective Role of Decorin in Primary Hepatocellular Carcinoma. Front. Oncol. 10:645. doi: 10.3389/fonc.2020.00645

Roden, L., and Smith, R. (1966). Structure of the neutral trisaccharide of the chondroitin 4-sulfate-protein linkage region. J. Biol. Chem. 241, 5949-5954. doi: 10.1016/s0021-9258(18)96362-3

Schjoldager, K. T., Narimatsu, Y., Joshi, H. J., and Clausen, H. (2020). Global view of human protein glycosylation pathways and functions. Nat. Rev. Mol. Cell Biol. 21, 729-749. doi: 10.1038/s41580-020-00294-x

Schneider, M., Al-Shareffi, E., and Haltiwanger, R. S. (2017). Biological functions of fucose in mammals. Glycobiology 27, 601-618. doi: 10.1093/glycob/c wx034

Shatton, J., and Schubert, M. (1954). Isolation of a mucoprotein from cartilage. J. Biol. Chem. 211, 565-573. doi: 10.1016/s0021-9258(18) 71147-2

Sugahara, K., Yamashina, I., De Waard, P., Van Halbeek, H., and Vliegenthart, J. F. (1988). Structural studies on sulfated glycopeptides from the carbohydrateprotein linkage region of chondroitin 4-sulfate proteoglycans of swarm rat chondrosarcoma. Demonstration of the structure Gal(4-O-sulfate)beta 1-3Gal beta 1-4XYL beta 1-O-Ser. J. Biol. Chem. 263, 10168-10174. doi: 10.1016/ s0021-9258(19)81492-8

Sugiura, N., Clausen, T. M., Shioiri, T., Gustavsson, T., Watanabe, H., and Salanti, A. (2016). Molecular dissection of placental malaria protein VAR2CSA interaction with a chemo-enzymatically synthesized chondroitin sulfate library. Glycoconj. J. 33, 985-994. doi: 10.1007/s10719-016-9685-z

Takemura, M., Noborn, F., Nilsson, J., Bowden, N., Nakato, E., Baker, S., et al. (2020). Chondroitin sulfate proteoglycan Windpipe modulates Hedgehog signaling in Drosophila. Mol. Biol. Cell 31, 813-824. doi: 10.1091/mbc.e19-060327

Thaysen-Andersen, M., Packer, N. H., and Schulz, B. L. (2016). Maturing Glycoproteomics Technologies Provide Unique Structural Insights into the N-glycoproteome and Its Regulation in Health and Disease. Mol. Cell Proteomics 15, 1773-1790. doi: $10.1074 / \mathrm{mcp} .0115 .0$ 57638

Toledo, A. G., Pihl, J., Spliid, C. B., Persson, A., Nilsson, J., Pereira, M. A., et al. (2020). An Affinity-Chromatography and Glycoproteomics Workflow to Profile the Chondroitin Sulfate Proteoglycans that interact with malarial 
VAR2CSA in the Placenta and in Cancer. Glycobiology 30, 989-1002. doi: 10. 1093/glycob/cwaa039

Tomlinson, A., Semblat, J. P., Gamain, B., and Chene, A. (2020). VAR2CSA-Mediated Host Defense Evasion of Plasmodium falciparum Infected Erythrocytes in Placental Malaria. Front. Immunol. 11:624126. doi: $10.3389 /$ fimmu.2020.624126

Wang, T. T., and Ravetch, J. V. (2019). Functional diversification of IgGs through Fc glycosylation. J. Clin. Invest. 129, 3492-3498. doi: 10.1172/jci 130029

Wen, J., Xiao, J., Rahdar, M., Choudhury, B. P., Cui, J., Taylor, G. S., et al. (2014). Xylose phosphorylation functions as a molecular switch to regulate proteoglycan biosynthesis. Proc. Natl. Acad. Sci. U. S. A. 111, 15723-15728.

Zhang, P., Lu, H., Peixoto, R. T., Pines, M. K., Ge, Y., Oku, S., et al. (2018). Heparan Sulfate Organizes Neuronal Synapses through Neurexin Partnerships. Cell 174, 1450-1464.e23.

Zhu, F., Li, D., and Chen, K. (2019). Structures and functions of invertebrate glycosylation. Open Biol. 9:180232.
Conflict of Interest: The authors declare that the research was conducted in the absence of any commercial or financial relationships that could be construed as a potential conflict of interest.

Publisher's Note: All claims expressed in this article are solely those of the authors and do not necessarily represent those of their affiliated organizations, or those of the publisher, the editors and the reviewers. Any product that may be evaluated in this article, or claim that may be made by its manufacturer, is not guaranteed or endorsed by the publisher.

Copyright $\odot 2021$ Noborn, Nikpour, Persson, Nilsson and Larson. This is an openaccess article distributed under the terms of the Creative Commons Attribution License (CC BY). The use, distribution or reproduction in other forums is permitted, provided the original author(s) and the copyright owner(s) are credited and that the original publication in this journal is cited, in accordance with accepted academic practice. No use, distribution or reproduction is permitted which does not comply with these terms. 